\title{
Illumination compensation for document images using local-global block analysis
}

\begin{abstract}
This paper presents the illumination compensation technique for document images using local-global block analysis. Imbalance illumination will affect the performance of classification and segmentation process because the darker regions conceal the information of the image. This method will split the image into non-overlapped blocks, and utilize the information within the local and global area of the image. The output images were binarized with simple global thresholding technique and the result shows that the output image is comparable in quality with the existed method. A comparative result will be presented with other document binarization methods.
\end{abstract}

Keyword: Illumination compensation; Local-global block analysis; OCR 